PHYSICAL REVIEW E 66, 046130 (2002)

\title{
Stationary correlations for a far-from-equilibrium spin chain
}

\author{
B. Schmittmann ${ }^{1}$ and F. Schmüser ${ }^{2}$ \\ ${ }^{1}$ Center for Stochastic Processes in Science and Engineering and Department of Physics, Virginia Tech, Blacksburg, \\ Virginia 24061-0435 \\ ${ }^{2}$ MPI für Physik komplexer Systeme, Nöthnitzer Strasse 38, 01187 Dresden, Germany
}

(Received 13 June 2002; published 24 October 2002)

\begin{abstract}
A kinetic one-dimensional Ising model on a ring evolves according to a generalization of Glauber rates, such that spins at even (odd) lattice sites experience a temperature $T_{e}\left(T_{o}\right)$. Detailed balance is violated so that the spin chain settles into a nonequilibrium stationary state, characterized by multiple interactions of increasing range and spin order. We derive the equations of motion for arbitrary correlation functions and solve them to obtain an exact representation of the steady state. Two nontrivial amplitudes reflect the sublattice symmetries; otherwise, correlations decay exponentially, modulo the periodicity of the ring. In the long-chain limit, they factorize into products of two-point functions, in precise analogy to the equilibrium Ising chain. The exact solution confirms the expectation, based on simulations and renormalization group arguments, that the longtime, long-distance behavior of this two-temperature model is Ising-like, in spite of the apparent complexity of the stationary distribution.
\end{abstract}

DOI: 10.1103/PhysRevE.66.046130

PACS number(s): 05.70.Ln, 05.50.+q, 75.10.Hk

\section{INTRODUCTION}

Exact solutions of simple model systems play a key role in statistical mechanics. They provide reliable quantitative information about regular as well as singular behavior, and serve as proving grounds for approximation schemes. Moreover, they can set important pointers in areas where a general theoretical framework is still lacking. A prime example is the study of nonequilibrium stationary states (NESS). Similar to systems in thermal equilibrium, NESS are characterized by time-independent macroscopic observables; however, at present these can only be computed with explicit reference to the imposed dynamics. There is, as yet, no equivalent of the Gibbs ensemble theory for far-from-equilibrium steady states. As a consequence, most progress to date is made by studying specific models.

Since the Ising model [1] is one of the most intimately known interacting many-particle systems, many nonequilibrium models depart from it by imposing an external force that drives the system out of equilibrium. Examples include couplings to multiple heat baths [2-4], competing Glauber and Kawasaki dynamics [5], or a current-inducing global bias [6]. In all of these models, reviewed in Ref. [7], the nonequilibrium perturbation violates the detailed balance symmetry of the equilibrium dynamics. On large time and length scales, one can probe how this affects universal behavior, or, adopting a more microscopic but no less fundamental perspective, one can ask how nonuniversal properties, such as the exact configurational probabilities, are modified. One finds, generically, that nonequilibrium forces have especially profound effects if (i) they couple to the bulk rather than the boundaries [8,9] of the system [10], and (ii) the dynamics satisfies a conservation law [11-13]. For example, Ising lattice gases with conserved particle number can be driven into two distinct nonequilibrium universality classes, depending on the symmetries of the external forces $[6,11]$. In contrast, Ising-like systems with nonconserved dynamics remain in the universality class of the equilibrium
Ising model, even if the usual $\mathbb{Z}_{2}$ symmetry of the Ising model is broken [14]. Even then, however, the violation of detailed balance leads to fundamental changes in the configurational probabilities, which must be identified and interpreted before we may hope to formalize our understanding of NESS. It is here that exact solutions are expected to be most helpful.

Motivated by these considerations, we recently [15] investigated a very simple nonequilibrium Ising-like model, namely, a one-dimensional interacting spin chain with spinflip dynamics coupled to two temperature baths. The rates are a simple, but nontrivial generalization of the familiar Glauber [16] rates: spins at odd (even) sites are coupled to a temperature $T_{o}\left(T_{e}\right)$. In two dimensions, this model $[3,4]$ exhibits an order-disorder phase transition that belongs to the Ising class, according to renormalization group arguments [14] and Monte Carlo simulations [4]. In one dimension, the two-spin correlation function can be calculated exactly [17]. Its only singularity lies at $T_{o}=T_{e}=0$, so that the lower critical dimension is $d=1$. Seeking an expression for the steady state, we solved the master equation perturbatively, in an expansion in the temperature difference of the two heat baths, up to and including second-order terms [15]. To our surprise, the full stationary distribution turned out to be rather complex. At each order, additional spin operators appear, characterized by longer spatial range interactions and higher-order spin products, and lower-order coupling constants acquire corrections. So, at first order, one encounters next-nearest-neighbor pair interactions, while at second order, two new terms appear: a next-next-nearest-neighbor pair interaction and a four-spin interaction spanning four nearestneighbor sites. All of these are allowed by symmetry, and none allowed by symmetry are absent. Given this structure, one can, at least in principle, extrapolate to higher orders in perturbation theory.

Of course, these findings immediately raise an obvious question: how does this relatively complicated stationary distribution generate long-wavelength behavior in the Ising uni- 
versality class? In this paper, we pursue an alternate route towards the answer. Instead of aiming for the stationary state directly, we seek its representation in terms of correlation functions-since the knowledge of all stationary correlation functions is equivalent to knowing the steady state. Starting from the master equation, we derive a hierarchy of equations of motion for correlation functions of arbitrary numbers of spins. In $d=1$, this hierarchy is closed and soluble. Remarkably, we find that - in contrast to the apparent complexity of the stationary distribution itself-the correlation functions are very simple. Apart from two nontrivial amplitudes that reflect the temperature difference between the two sublattices, the structure of correlations is completely analogous to the equilibrium Ising model. Specifically, for $N \rightarrow \infty$, arbitrary (even) $m$-point correlation functions factorize into a product of $m / 2$ two-point correlations. Of course, all correlation functions involving an odd number of spins vanish by symmetry.

While some exact analytic results for steady-state distributions are available, they are confined to three classes of systems. First, one-dimensional lattice gas models, restricted to excluded volume interactions, such as the asymmetric exclusion process and its relatives [18-20]; second, very special one-dimensional spin systems whose master equations are solved by the Ising Boltzmann factor $[6,21,22]$; and third, interacting systems in one or two dimensions on very small lattices so that the number of degrees of freedom remains manageable [23]. To the best of our knowledge, the work presented here is amongst the first complete solutions for nonequilibrium stationary states with nontrivial (nearestneighbor) interactions and arbitrary number of degrees of freedom.

The paper is organized as follows. We first introduce our model and its master equation. Next, we derive the equations of motion for arbitrary correlation functions. Following a brief review of the solution [17] for the two-point correlation function, we show how four-point correlations can be factorized into two-point correlations in the long chain $(N \rightarrow \infty)$ limit. We then postulate that all correlation functions factorize in this manner, and show that this factorization solves the equations of motion. In the Appendix, we generalize this solution to finite systems with periodic boundary conditions. We conclude with some comments and open questions.

\section{THE MODEL}

Our model is defined on a one-dimensional ring, with an even number $N$ of sites, and periodic boundary conditions. A spin variable, $\sigma_{i}= \pm 1$, denotes the value of the spin at site $i$, and nearest-neighbor spins interact according to the usual Ising Hamiltonian

$$
\mathcal{H}=-J \sum_{i} \sigma_{i} \sigma_{i+1}
$$

with an exchange coupling $J$. The dynamics is a nonequilibrium generalization of the usual Glauber model [16]: spins on even and odd lattice sites experience different temperatures, $T_{e}$ and $T_{o}$. Specifically, a configuration $\{\sigma\}$ $=\left\{\sigma_{1}, \sigma_{2}, \ldots, \sigma_{N}\right\}$ evolves into a new configuration by flipping a randomly selected spin $\sigma_{i}$ with a rate [17]

$$
w_{i}\left(\sigma_{i} \rightarrow-\sigma_{i}\right)=1-\frac{\gamma_{i}}{2} \sigma_{i}\left(\sigma_{i-1}+\sigma_{i+1}\right),
$$

where

$$
\gamma_{i}=\left\{\begin{array}{lll}
\gamma_{e}=\tanh \left(2 J / k_{B} T_{e}\right), & i \text { even } \\
\gamma_{o}=\tanh \left(2 J / k_{B} T_{o}\right), & i \text { odd. }
\end{array}\right.
$$

Thus, the full time-dependent configurational probability $p(\{\sigma\} ; t)$ evolves according to a master equation,

$$
\begin{aligned}
\partial_{t} p(\{\sigma\} ; t)= & \sum_{i=1}^{N}\left[-w_{i}\left(\sigma_{i} \rightarrow-\sigma_{i}\right) p(\{\sigma\} ; t)\right. \\
& \left.+w_{i}\left(-\sigma_{i} \rightarrow \sigma_{i}\right) p\left(\left\{\sigma^{[i]}\right\} ; t\right)\right],
\end{aligned}
$$

where $\left\{\sigma^{[i]}\right\}$ differs from $\{\sigma\}$ by a flip of the $i$ th spin. A trivial time scale has been set to unity, and we use dimensionless units for inverse temperature, i.e., $\beta_{e} \equiv J /\left(k_{B} T_{e}\right)$, etc.

Our goal in the following is to find a representation for the stationary solution of Eq. (4), $q(\{\sigma\})$ $\equiv \lim _{t \rightarrow \infty} p(\{\sigma\} ; t)$. This limit is unique, since Eq. (4) is ergodic: every configuration $\{\sigma\}$ can be reached in finite time from every other configuration $\left\{\sigma^{\prime}\right\}$ (unless $T_{e}=T_{o}=0$ ). For equal temperatures $T \equiv T_{e}=T_{o}$, the steady-state is just the (canonical) distribution for the Ising chain,

$$
q_{o}(\{\sigma\})=\frac{1}{Z} \exp \left(-H / k_{B} T\right) .
$$

It is of course straightforward to compute arbitrary correlation functions for the equilibrium Ising chain. Since the Ising model is invariant under a global spin flip $\left(\mathbb{Z}_{2}\right.$ symmetry), only correlations of even numbers of spins are nonzero, and are easily expressed in terms of the parameter $\bar{\omega}$ $=\tanh \left(J / k_{B} T\right)$. Of course, we may-and always will—order the arguments of an $m$-point correlation function ( $m$ even) without loss of generality, such that $1 \leqslant k_{1}<k_{2}<\cdots<k_{m}$ $\leqslant N$. Then, in the $N \rightarrow \infty$ limit one finds easily,

$$
\begin{aligned}
\left\langle\sigma_{k_{1}} \cdots \sigma_{k_{m}}\right\rangle^{e q} & =\bar{\omega}^{\left(k_{2}-k_{1}\right)+\left(k_{4}-k_{3}\right)+\cdots+\left(k_{m}-k_{m-1}\right)} \\
& =\left\langle\sigma_{k_{1}} \sigma_{k_{2}}\right\rangle^{e q}\left\langle\sigma_{k_{3}} \sigma_{k_{4}}\right\rangle^{e q} \cdots\left\langle\sigma_{k_{m-1}} \sigma_{k_{m}}\right\rangle^{e q}
\end{aligned}
$$$$
\text { for } m \text { even and } N \rightarrow \infty \text {, }
$$

i.e., a general correlation function factorizes into a product of two-point functions.

We now turn to the nonequilibrium model, characterized by different temperatures $T_{e} \neq T_{o}$. The associated stationary state violates detailed balance [15] and differs from the Boltzmann distribution, Eq. (5). The degree to which detailed balance is violated can be measured by the parameter $d$ $\equiv\left(\gamma_{o}-\gamma_{e}\right) / 2$. Similar to the Ising model, the stationary state is invariant under a global spin flip $\left(\mathbb{Z}_{2}\right)$. As a consequence, 
all stationary correlations of an odd number of spins vanish identically, and again, only even correlations need to be discussed. We also note the symmetry under translations by $n$ lattice sites, $T_{n}$, combined with $d \rightarrow-d$ if $n$ is odd:

$$
\begin{gathered}
q(\{\sigma\} ; d)=q(\{-\sigma\} ; d), \\
q(\{\sigma\} ; d)=q\left(\mathbb{T}_{n}\{\sigma\} ;(-1)^{n} d\right) .
\end{gathered}
$$

While a direct exact solution of the master equation has proven elusive, a perturbative calculation, in powers of $d$, shows [15] that the stationary distribution for this nonequilibrium model is rather complicated, with longer-range and higher-order spin operators appearing. Specifically, writing $q(\{\sigma\}) \equiv \widetilde{Z}^{-1} \exp [V(\{\sigma\})]$, we find that the potential function $V(\{\sigma\})$, to second order in $d$, has the form

$$
\begin{aligned}
V(\{\sigma\})= & \bar{\beta} \sum_{i} \sigma_{i} \sigma_{i+1}+d \lambda \sum_{i}(-1)^{i} \sigma_{i} \sigma_{i+2} \\
& +2(d \lambda)^{2} \sum_{i}\left[\sigma_{i} \sigma_{i+2}+\sigma_{i} \sigma_{i+1} \sigma_{i+2} \sigma_{i+3}\right. \\
& \left.-\operatorname{coth}(2 \bar{\beta})\left(\sigma_{i} \sigma_{i+1}+\sigma_{i} \sigma_{i+3}\right)\right],
\end{aligned}
$$

where $\tanh (2 \bar{\beta}) \equiv\left(\gamma_{e}+\gamma_{o}\right) / 2$ and $\lambda=-\frac{1}{8} \sinh (4 \bar{\beta})$. An analysis of the structure of the perturbation series indicates that, at each order, additional spin operators appear, consisting of spatially longer-range interactions and higher-order spin products. It is therefore quite remarkable that the correlation functions turn out to be very similar to those of the Ising model, as we now proceed to show.

\section{EQUATIONS OF MOTION FOR ARBITRARY CORRELATION FUNCTIONS}

We begin by deriving the equations of motion for arbitrary time-dependent $m$-point spin correlation functions, $\left\langle\sigma_{k_{1}} \sigma_{k_{2}} \cdots \sigma_{k_{m}}\right\rangle_{t}$, starting from the master equation:

$$
\begin{aligned}
\partial_{t}\left\langle\sigma_{k_{1}} \sigma_{k_{2}} \cdots \sigma_{k_{m}}\right\rangle_{t} & \\
= & \sum_{i=1}^{N}\left\{\sum _ { \{ \sigma \} } \sigma _ { k _ { 1 } } \sigma _ { k _ { 2 } } \cdots \sigma _ { k _ { m } } \left[-w_{i}\left(\sigma_{i} \rightarrow-\sigma_{i}\right) p(\{\sigma\} ; t)\right.\right. \\
& \left.\left.+w_{i}\left(-\sigma_{i} \rightarrow \sigma_{i}\right) p\left(\left\{\sigma^{[i]}\right\} ; t\right)\right]\right\} .
\end{aligned}
$$

Here, the subscript $\langle 0\rangle_{t}$ will be used to distinguish the timedependent averages from their stationary limits, $\langle 0\rangle$ $\equiv \lim _{t \rightarrow \infty}\langle\rangle_{t}$. Due to the sum over all configurations, the \{\} bracket on the right-hand side obviously vanishes for all sites $i$, which do not belong to the set $\left\{k_{1}, k_{2}, \ldots, k_{m}\right\}$, and one finds easily that

$$
\begin{aligned}
& \partial_{t}\left\langle\sigma_{k_{1}} \sigma_{k_{2}} \cdots \sigma_{k_{m}}\right\rangle_{t} \\
& \quad=-2 \sum_{i=1}^{m}\left\langle\sigma_{k_{1}} \sigma_{k_{2}} \cdots \sigma_{k_{m}} w_{k_{i}}\left(\sigma_{k_{i}} \rightarrow-\sigma_{k_{i}}\right)\right\rangle_{t} .
\end{aligned}
$$

Inserting Eq. (2) for $w_{k_{i}}\left(\sigma_{k_{i}} \rightarrow-\sigma_{k_{i}}\right)$ and taking the infinite time limit, we obtain the equations satisfied by the stationary correlation functions, $\lim _{t \rightarrow \infty}\left\langle\sigma_{k_{1}} \sigma_{k_{2}} \cdots \sigma_{k_{m}}\right\rangle_{t}$ $\begin{aligned} \equiv\left\langle\sigma_{k_{1}} \sigma_{k_{2}} \cdots \sigma_{k_{m}}\right\rangle: & \\ 0= & -2 m\left\langle\sigma_{k_{1}} \sigma_{k_{2}} \cdots \sigma_{k_{m}}\right\rangle \\ & +\sum_{i=1}^{m} \gamma_{k_{i}}\left\langle\sigma_{k_{1}} \cdots \sigma_{k_{i-1}}\left(\sigma_{k_{i}+1}+\sigma_{k_{i}-1}\right) \sigma_{k_{i+1}} \cdots \sigma_{k_{m}}\right\rangle .\end{aligned}$

As mentioned before, all stationary correlations of odd numbers of spins are trivially zero, and we need to focus only on the case of even $m$. Moreover, all stationary correlations are translationally invariant, modulo the sublattice structure. As in the Glauber model [24], the hierarchy of correlation functions is closed; the equation for $m$-point correlations does not involve any higher correlations, and it is homogeneous, provided there are no nearest-neighbor pairs among the arguments. Otherwise, the $m$-point correlations couple to lower correlation functions, which appear as inhomogeneities. In the following section, we motivate an ansatz for $\left\langle\sigma_{k_{1}} \sigma_{k_{2}} \cdots \sigma_{k_{m}}\right\rangle$, and then show that it satisfies Eq. (9) for any choice of $\left\{k_{1}, k_{2}, \ldots, k_{m}\right\}$.

\section{EXACT SOLUTIONS FOR STATIONARY CORRELATIONS}

To establish several key relations, we briefly review the exact solution for the two-point functions $\left\langle\sigma_{i} \sigma_{j}\right\rangle$ [17]. For this case, Eq. (9) reads

$$
\begin{aligned}
0= & -4\left\langle\sigma_{i} \sigma_{j}\right\rangle+\gamma_{i}\left\langle\left(\sigma_{i+1}+\sigma_{i-1}\right) \sigma_{j}\right\rangle \\
& +\gamma_{j}\left\langle\sigma_{i}\left(\sigma_{j+1}+\sigma_{j-1}\right)\right\rangle .
\end{aligned}
$$

For nearest-neighbor sites, e.g., $j=i+1$, this equation becomes inhomogeneous:

$$
\begin{aligned}
0= & -4\left\langle\sigma_{i} \sigma_{i+1}\right\rangle+\gamma_{i}\left\langle\sigma_{i-1} \sigma_{i+1}\right\rangle+\gamma_{i+1}\left\langle\sigma_{i} \sigma_{i+2}\right\rangle+\gamma_{i} \\
& +\gamma_{i+1} .
\end{aligned}
$$

For simplicity, we restrict the discussion in this section to correlation functions in the thermodynamic limit $N \rightarrow \infty$. Thus we may label the lattice sites by the integers, $i \in \mathbb{Z}$. Correlations on finite periodic chains and some details for the $N \rightarrow \infty$ limit will be addressed in the Appendix. Following Ref. [17], it is easy to show that the solution is unique and takes the form (for $i<j$, without loss of generality)

$$
\left\langle\sigma_{i} \sigma_{j}\right\rangle=\sqrt{A_{i} A_{j}} \omega^{j-i},
$$

with the spatial decay length controlled by the parameter

$$
\omega=\frac{1}{\sqrt{\gamma_{e} \gamma_{o}}}\left(1-\sqrt{1-\gamma_{e} \gamma_{o}}\right) .
$$


The key difference to the equilibrium Ising model is the emergence of two amplitudes, to match the sublattice symmetries, namely,

$$
A_{i} \equiv \begin{cases}A_{e}=\left(\gamma_{e}+\gamma_{o}\right) /\left(2 \gamma_{o}\right), & i \text { even } \\ A_{o}=\left(\gamma_{e}+\gamma_{o}\right) /\left(2 \gamma_{e}\right), & i \text { odd. }\end{cases}
$$

For later reference, we note that these relations imply a further identity for products of pair correlations, namely, for any $i<j<j+1<l$,

$$
\begin{aligned}
0= & \left(\gamma_{j}+\gamma_{j+1}\right)\left\langle\sigma_{i} \sigma_{l}\right\rangle-\gamma_{j}\left\langle\sigma_{i} \sigma_{j+1}\right\rangle\left\langle\sigma_{j+1} \sigma_{l}\right\rangle \\
& -\gamma_{j+1}\left\langle\sigma_{i} \sigma_{j}\right\rangle\left\langle\sigma_{j} \sigma_{l}\right\rangle .
\end{aligned}
$$

This relation will be needed in the discussion of four-point functions.

It is quite remarkable that the three quantities $\omega, A_{e}$, and $A_{o}$ also determine all higher correlation functions, through a structure that is almost perfectly analogous to the equilibrium case. Deferring the case of finite $N$ to the Appendix, we consider only an infinite chain here. Focusing on even correlations, we assert that arbitrary $m$-point correlation functions, with $k_{1}<k_{2}<\cdots<k_{m}$ and $m$ even, are given by

$$
\begin{aligned}
& \left\langle\sigma_{k_{1}} \cdots \sigma_{k_{m}}\right\rangle \\
& \quad=\sqrt{A_{k_{1}} A_{k_{2}} \cdots A_{k_{m}}} \omega^{\left(k_{2}-k_{1}\right)+\left(k_{4}-k_{3}\right)+\cdots+\left(k_{m}-k_{m-1}\right)} \\
& =\left\langle\sigma_{k_{1}} \sigma_{k_{2}}\right\rangle\left\langle\sigma_{k_{3}} \sigma_{k_{4}}\right\rangle \cdots\left\langle\sigma_{k_{m-1}} \sigma_{k_{m}}\right\rangle
\end{aligned}
$$

i.e., higher correlation functions factorize into two-spin correlations as in the equilibrium case, Eq. (6). However, two features distinguish these correlations from their equilibrium counterparts. First, the spatial dependence is controlled by a different parameter, $\omega$. In analogy to $\bar{\omega}$ for the equilibrium system, $\omega$ defines an effective temperature for the nonequilibrium system, via $\omega \equiv \tanh \left(J / k_{B} T_{\text {eff }}\right)$. $T_{\text {eff }}$ diverges with $T_{o}$ or $T_{e}$ but vanishes only if both $T_{o}$ and $T_{e}$ go to zero. As a result, the correlation length $\xi$, defined via $\ln \omega \equiv-\xi^{-1}$, diverges only if both temperatures vanish. Second, and more importantly, we note the appearance of the even/odd amplitudes $A_{e}, A_{o}$, reflecting the sublattice identities of the two spins. These amplitudes carry the primary information about the nonequilibrium nature of our dynamics.

Before turning to a general proof of Eq. (16), it is instructive to confirm it explicitly for the four-point functions. In this case, factorization implies

$$
\left\langle\sigma_{i} \sigma_{j} \sigma_{k} \sigma_{l}\right\rangle=\left\langle\sigma_{i} \sigma_{j}\right\rangle\left\langle\sigma_{k} \sigma_{l}\right\rangle
$$

for $i<j<k<l$. When inserting this ansatz into the righthand side of Eq. (9), we need to distinguish whether $j$ and $k$ are nearest-neighbor sites or not. If $k>j+1$, we obtain

$$
\begin{aligned}
&-8\left\langle\sigma_{i} \sigma_{j} \sigma_{k} \sigma_{l}\right\rangle+\gamma_{i}\left\langle\left(\sigma_{i+1}+\sigma_{i-1}\right) \sigma_{j} \sigma_{k} \sigma_{l}\right\rangle \\
&+ \gamma_{j}\left\langle\sigma_{i}\left(\sigma_{j+1}+\sigma_{j-1}\right) \sigma_{k} \sigma_{l}\right\rangle \\
&+ \gamma_{k}\left\langle\sigma_{i} \sigma_{j}\left(\sigma_{k+1}+\sigma_{k-1}\right) \sigma_{l}\right\rangle+\gamma_{l}\left\langle\sigma_{i} \sigma_{j} \sigma_{k}\left(\sigma_{l+1}+\sigma_{l-1}\right)\right\rangle \\
&= {\left[-4\left\langle\sigma_{i} \sigma_{j}\right\rangle+\gamma_{i}\left\langle\left(\sigma_{i+1}+\sigma_{i-1}\right) \sigma_{j}\right\rangle\right.} \\
&\left.+\gamma_{j}\left\langle\sigma_{i}\left(\sigma_{j+1}+\sigma_{j-1}\right)\right\rangle\right]\left\langle\sigma_{k} \sigma_{l}\right\rangle+\left[-4\left\langle\sigma_{k} \sigma_{l}\right\rangle\right. \\
&\left.+\gamma_{k}\left\langle\left(\sigma_{k+1}+\sigma_{k-1}\right) \sigma_{l}\right\rangle+\gamma_{l}\left\langle\sigma_{j} \sigma_{k}\left(\sigma_{l+1}+\sigma_{l-1}\right)\right\rangle\right] \\
& \times\left\langle\sigma_{i} \sigma_{j}\right\rangle=0,
\end{aligned}
$$

since the expressions in the square brackets vanish for the stationary two-point functions, by virtue of Eq. (10) or (11), depending on whether $(i, j)$ or $(k, l)$ are nearest neighbors or not. If $k=j+1$, we need to consider a slightly different equation, namely,

$$
\begin{aligned}
-8 & \left\langle\sigma_{i} \sigma_{j} \sigma_{j+1} \sigma_{l}\right\rangle+\gamma_{i}\left\langle\left(\sigma_{i+1}+\sigma_{i-1}\right) \sigma_{j} \sigma_{j+1} \sigma_{l}\right\rangle \\
& +\gamma_{j}\left\langle\sigma_{i}\left(\sigma_{j+1}+\sigma_{j-1}\right) \sigma_{j+1} \sigma_{l}\right\rangle \\
& +\gamma_{j+1}\left\langle\sigma_{i} \sigma_{j}\left(\sigma_{j+2}+\sigma_{j}\right) \sigma_{l}\right\rangle+\gamma_{l}\left\langle\sigma _ { i } \sigma _ { j } \sigma _ { j + 1 } \left(\sigma_{l+1}\right.\right. \\
& \left.\left.+\sigma_{l-1}\right)\right\rangle=\left[-4\left\langle\sigma_{i} \sigma_{j}\right\rangle+\gamma_{i}\left\langle\left(\sigma_{i+1}+\sigma_{i-1}\right) \sigma_{j}\right\rangle\right. \\
& \left.+\gamma_{j}\left\langle\sigma_{i} \sigma_{j-1}\right\rangle\right]\left\langle\sigma_{j+1} \sigma_{l}\right\rangle+\gamma_{j}\left\langle\sigma_{i} \sigma_{l}\right\rangle+\left\langle\sigma_{i} \sigma_{j}\right\rangle \\
& \times\left[-4\left\langle\sigma_{j+1} \sigma_{l}\right\rangle+\gamma_{j+1}\left\langle\sigma_{j+2} \sigma_{l}\right\rangle+\gamma_{l}\left\langle\sigma _ { j + 1 } \left(\sigma_{l+1}\right.\right.\right. \\
& \left.\left.\left.+\sigma_{l-1}\right)\right\rangle\right]+\gamma_{j+1}\left\langle\sigma_{i} \sigma_{l}\right\rangle=\gamma_{j}\left\langle\sigma_{i} \sigma_{l}\right\rangle+\gamma_{j+1}\left\langle\sigma_{i} \sigma_{l}\right\rangle \\
& -\gamma_{j}\left\langle\sigma_{i} \sigma_{j+1}\right\rangle\left\langle\sigma_{j+1} \sigma_{l}\right\rangle-\gamma_{j+1}\left\langle\sigma_{i} \sigma_{j}\right\rangle\left\langle\sigma_{j} \sigma_{l}\right\rangle=0 .
\end{aligned}
$$

The last equality follows from Eq. (15).

The proof is completed by induction. Assuming that the factorization has been proven for $(m-2)$-point functions, it is sufficient to show that the ansatz

$$
\left\langle\sigma_{k_{1}} \cdots \sigma_{k_{m}}\right\rangle=\left\langle\sigma_{k_{1}} \cdots \sigma_{k_{m-2}}\right\rangle\left\langle\sigma_{k_{m-1}} \sigma_{k_{m}}\right\rangle
$$

solves the equations of motion for the $m$-point functions. Again, we need to distinguish whether $k_{m-2}$ and $k_{m-1}$ are nearest neighbors or not. If they are separated by more than one lattice spacing, i.e., if $k_{m-1}>k_{m-2}+1$, we have

$$
\begin{aligned}
-2 m & \left\langle\sigma_{k_{1}} \sigma_{k_{2}} \cdots \sigma_{k_{m}}\right\rangle+\sum_{i=1}^{m} \gamma_{k_{i}}\left\langle\sigma_{k_{1}} \cdots \sigma_{k_{i-1}}\left(\sigma_{k_{i}+1}+\sigma_{k_{i}-1}\right) \sigma_{k_{i+1}} \cdots \sigma_{k_{m}}\right\rangle \\
= & \left\langle\sigma_{k_{m-1}} \sigma_{k_{m}}\right\rangle\left[-2(m-2)\left\langle\sigma_{k_{1}} \cdots \sigma_{k_{m-2}}\right\rangle+\sum_{i=1}^{m-2} \gamma_{k_{i}}\left\langle\sigma_{k_{1}} \cdots \sigma_{k_{i-1}}\left(\sigma_{k_{i}+1}+\sigma_{k_{i}-1}\right) \sigma_{k_{i+1}} \cdots \sigma_{k_{m-2}}\right\rangle\right] \\
& +\left\langle\sigma_{k_{1}} \cdots \sigma_{k_{m-2}}\right\rangle\left[-2\left\langle\sigma_{k_{m-1}} \sigma_{k_{m}}\right\rangle+\gamma_{k_{m-1}}\left\langle\left(\sigma_{k_{m-1}+1}+\sigma_{k_{m-1}-1}\right) \sigma_{k_{m}}\right\rangle+\gamma_{k_{m}}\left\langle\sigma_{k_{m-1}}\left(\sigma_{k_{m}+1}+\sigma_{k_{m}-1}\right)\right\rangle\right]=0 .
\end{aligned}
$$

Here, we have used the fact that both square brackets vanish, since they enclose the equations of motion for the two- and 
( $m-2)$-point functions, respectively. Next, we consider the case $k_{m-1}=k_{m-2}+1$. Again, we add and subtract the terms missing from the full equations of motion of the lower correlations functions:

$$
\begin{aligned}
& -2 m\left\langle\sigma_{k_{1}} \sigma_{k_{2}} \cdots \sigma_{k_{m}}\right\rangle+\sum_{i=1}^{m} \gamma_{k_{i}}\left\langle\sigma_{k_{1}} \cdots \sigma_{k_{i-1}}\left(\sigma_{k_{i}+1}+\sigma_{k_{i}-1}\right) \sigma_{k_{i+1}} \cdots \sigma_{k_{m}}\right\rangle \\
& =-\gamma_{k_{m-2}}\left\langle\sigma_{k_{1}} \cdots \sigma_{k_{m-3}} \sigma_{k_{m-2}+1}\right\rangle\left\langle\sigma_{k_{m-1}} \sigma_{k_{m}}\right\rangle+\gamma_{k_{m-2}}\left\langle\sigma_{k_{1}} \cdots \sigma_{k_{m-3}} \sigma_{k_{m}}\right\rangle-\gamma_{k_{m-1}}\left\langle\sigma_{k_{1}} \cdots \sigma_{k_{m-2}}\right\rangle\left\langle\sigma_{k_{m-1}-1} \sigma_{k_{m}}\right\rangle \\
& \quad+\gamma_{k_{m-1}}\left\langle\sigma_{k_{1}} \cdots \sigma_{k_{m-3}} \sigma_{k_{m}}\right\rangle=\left\langle\sigma_{k_{1}} \cdots \sigma_{k_{m-4}}\right\rangle\left\{\left(\gamma_{k_{m-2}}+\gamma_{k_{m-1}}\right)\left\langle\sigma_{k_{m-3}} \sigma_{k_{m}}\right\rangle-\gamma_{k_{m-2}}\left\langle\sigma_{k_{m-3}} \sigma_{k_{m-2}+1}\right\rangle\left\langle\sigma_{k_{m-1}} \sigma_{k_{m}}\right\rangle\right. \\
& \left.-\gamma_{k_{m-1}}\left\langle\sigma_{k_{m-3}} \sigma_{k_{m-2}}\right\rangle\left\langle\sigma_{k_{m-1}-1} \sigma_{k_{m}}\right\rangle\right\}=0 .
\end{aligned}
$$

To obtain the last two identities, we have factored out an $(m-4)$-point correlation, and used Eq. (15). This concludes the proof. We emphasize again that the condition $N \gg 1$ has been imposed for reasons of simplicity alone: expressions for arbitrary correlation functions on finite rings, while somewhat more cumbersome, are easily derived and also follow the Ising pattern, as we will show in the Appendix.

Finally, we discuss one special case of the twotemperature Glauber dynamics where the two-point correlations do not have the form of Eq. (12). This is the case if one of the two parameters $\gamma_{e}, \gamma_{o}$ is zero, the other finite, i.e., the temperature of one sublattice is infinite. Without loss of generality we assume $\gamma_{o}=0$ in the following. One can then easily show directly from the equation of motion (10) that the two-point function reads

$$
\left\langle\begin{array}{ll}
\sigma_{i} & \sigma_{j}
\end{array}\right\rangle \equiv\left\{\begin{array}{ll}
\gamma_{e} / 4 & \text { if } j-i=1 \\
\gamma_{e}^{2} / 8 & \text { if } j-i=2 \\
0 & \text { otherwise. }
\end{array} \text { and } i\right. \text { even }
$$

Hence, spins are only correlated over a distance of at most two lattice sites. Turning to the higher correlations, we observe that Eq. (15) also holds for these parameter values. Therefore, $m$-point correlations ( $m$ even) again factorize into two-point correlations.

\section{CONCLUSIONS}

To summarize, we have found an exact solution for all stationary correlation functions of a one-dimensional nonequilibrium Ising spin chain with an even number $N$ of sites. The system is globally coupled to two temperature baths: spins on odd (even) lattice sites experience a temperature $T_{o}$ $\left(T_{e}\right)$ and flip according to a generalization of the familiar Glauber rates. The presence of two different temperatures violates detailed balance and maintains a nontrivial nonequilibrium stationary state. The complete set of correlation functions provides us with the full, exact solution for this steady state. This allows us to reconcile two potentially contradictory earlier findings: while simulations and renormalization group arguments indicate that the long-wavelength behavior of nonconserved two-temperature models should be Isinglike, a perturbative analysis of the stationary distribution $q(\{\sigma\})$ showed that a large number of long-range and mul- tispin interactions are present in $\ln q(\{\sigma\})$. Here, we have shown that the exact correlation functions for this model are remarkably Ising-like: they decay exponentially with a characteristic correlation length, which diverges only if both $T_{o}$ and $T_{e}$ vanish. Also, in the $N \rightarrow \infty$ limit, arbitrary (even) $m$-point correlations factorize into products of two-point correlations, following exactly the same scheme in both the Ising and the two-temperature chain. The only key difference is the appearance of two nontrivial amplitudes, $A_{o}$ and $A_{e}$, which reflect the sublattice symmetry: for each spin on an odd (even) site, the correlation function carries a factor of $\sqrt{A_{o}}\left(\sqrt{A_{e}}\right)$. Of course, stationary correlations of odd numbers of spins vanish identically, due to symmetry. In short, the correlations of the model are entirely consistent with its Ising-like long-distance properties.

It is remarkable - and not at all immediately obviousthat these two amplitudes should be the only remnants of the large number of interactions in $q(\{\sigma\})$. There is, however, a very simple and elegant representation [25] of the stationary state in terms of an extended Ising model, consisting of $2 \mathrm{~N}$ spins on a comblike $d=1$ lattice with Hamiltonian $\mathcal{H}_{\text {aux }}=$ $-J \Sigma_{i} s_{i} s_{i+1}-J_{o} \Sigma_{\text {odd } i} s_{i} \sigma_{i}-J_{e} \Sigma_{\text {even } i} s_{i} \sigma_{i}$. Here, the spins $\left\{s_{i}\right\}$ form an auxiliary set that must be traced out in order to obtain stationary observables associated with the original variables $\left\{\sigma_{i}\right\}$. If the interactions $J, J_{o}$, and $J_{e}$ are tuned appropriately in the complex plane, the $\sigma$ correlations of $\mathcal{H}_{\text {aux }}$ are identical to those of the two-temperature model. Further, the exact stationary state of the two-temperature model follows as $q(\{\sigma\}) \propto \operatorname{Tr}_{\left\{s_{i}\right\}} \exp \left[-\mathcal{H}_{\text {aux }}\right]$. Details and generalizations will be presented elsewhere [26].

It would of course be interesting to investigate other nonequilibrium versions of Glauber dynamics. Will the correlations still have a rather simple structure if a spin chain is coupled, in a translation-invariant manner, to $n>2$ different temperatures? Will they still factorize, provided a sufficiently large number of amplitudes is introduced? Clearly, we still need to cover lots of ground before even such simple nonequilibrium systems are fully understood.

\section{ACKNOWLEDGMENTS}

We thank H. J. Hilhorst, R. K. P. Zia, J. Slawny, U. C. Täuber, W. Just, E. Ben-Naim, and M. Hastings for fruitful discussions. Financial support from the NSF through the Di- 
vision of Materials Research and from the DFG through Contract No. SCHM 1537 is gratefully acknowledged.

\section{APPENDIX: STATIONARY CORRELATIONS IN FINITE CHAINS}

In this appendix, we address the correlation functions for our model on a finite periodic chain (a ring) of $N$ sites, with $i=1,2, \ldots, N$. To set the scene, we first review the equilibrium case, with uniform temperature $T$. The correlation functions of the Ising model on a ring are well known. Using an ordered set of arguments, $1 \leqslant k_{1}<k_{2}<\cdots<k_{m} \leqslant N$ without loss of generality, one finds

$$
\begin{gathered}
\left\langle\sigma_{k_{1}} \cdots \sigma_{k_{m}}\right\rangle^{e q}=0 \text { for odd } m \\
\left\langle\sigma_{k_{1}} \cdots \sigma_{k_{m}}\right\rangle^{e q}= \\
\frac{1}{1+\bar{\omega}^{N}}\left\{\bar{\omega}^{\left(k_{2}-k_{1}\right)+\left(k_{4}-k_{3}\right)+\cdots+\left(k_{m}-k_{m-1}\right)}\right. \\
\left.+\bar{\omega}^{N-\left(k_{2}-k_{1}\right)-\left(k_{4}-k_{3}\right)-\cdots-\left(k_{m}-k_{m-1}\right)}\right\}
\end{gathered}
$$$$
\text { for even } m \text {, }
$$

with $\bar{\omega}=\tanh \left(J / k_{B} T\right)<1$. The periodicity of the ring implies that $\left\langle\sigma_{k_{1}} \cdots \sigma_{k_{m}}\right\rangle^{e q}$ is invariant under $\bar{\omega}^{n} \rightarrow \bar{\omega}^{N-n}$ for any integer $0 \leqslant n \leqslant N$. For $N \rightarrow \infty$, Eq. (A1) obviously reduces to Eq. (6).

Turning to the nonequilibrium case with two temperatures, we first assert that the stationary two-point correlation function, with $1 \leqslant i<j \leqslant N$, is given by

$$
\left\langle\sigma_{i} \sigma_{j}\right\rangle=\frac{1}{Z(\omega)} \sqrt{A_{i} A_{j}}\left[\omega^{j-i}+\omega^{N-(j-i)}\right],
$$

where the parameter $\omega$ and the amplitudes $A_{i} \in\left\{A_{e}, A_{o}\right\}$ are simply those of Eqs. (13) and (14), and the normalization factor $Z(\omega)$ is given by $Z(\omega)=1+\omega^{N}$. Clearly, $\left\langle\sigma_{i} \sigma_{j}\right\rangle$ is periodic with period $N$. For easy reference, we fill in some details here which are also relevant to the $N \rightarrow \infty$ case.

Equation (10) is essentially a second-order difference equation in the variable $j-i$. To find a unique solution, we need two boundary conditions: one of these is Eq. (11) for $j-i=1$ and the other is the requirement that for $\left\langle\sigma_{i} \sigma_{j}\right\rangle$ to be periodic with period $N$ (or, for $N \rightarrow \infty$, that $\left\langle\sigma_{i} \sigma_{j}\right\rangle$ vanish for large separations). We therefore expect a superposition of two linearly independent solutions whose coefficients can then be determined. Away from the boundaries, the ansatz $\left\langle\sigma_{i} \sigma_{j}\right\rangle=\sqrt{A_{i} A_{j}} \omega^{j-i}$ reduces Eq. (10) to three relations, for the combinations $i, j$ odd/odd, even/even, and odd/even, respectively,

$$
\begin{gathered}
0=-4 A_{o}+2 \gamma_{o} \sqrt{A_{e} A_{o}}\left(\omega+\omega^{-1}\right), \\
0=-4 A_{e}+2 \gamma_{e} \sqrt{A_{e} A_{o}}\left(\omega+\omega^{-1}\right), \\
0=-4 \sqrt{A_{e} A_{o}}+\left(\gamma_{o} A_{e}+\gamma_{e} A_{o}\right)\left(\omega+\omega^{-1}\right) .
\end{gathered}
$$

We note that the third equation is simply a linear combination of the first and second, expressing the fact that there are only two amplitudes, and not three, as one might have as- sumed (cf. Ref. [17]) based on the three types (odd/odd, even/even, odd/even) of pair correlations. Moreover, the symmetry under $\omega \leftrightarrow \omega^{-1}$ implies that, for each solution $\omega$, $\omega^{-1}$ is also a solution. Proceeding to solve the system, we find $\gamma_{e} A_{o}=\gamma_{o} A_{e}$ and

$$
\omega+\omega^{-1}=\frac{2}{\sqrt{\gamma_{o} \gamma_{e}}} \Rightarrow \omega_{ \pm}=\frac{1}{\sqrt{\gamma_{e} \gamma_{o}}}\left(1 \pm \sqrt{1-\gamma_{e} \gamma_{o}}\right)
$$

with $\omega_{+}=1 / \omega_{-}$. The two roots $\omega_{ \pm}$of this quadratic equation provide us with the two anticipated linearly independent solutions. However, to satisfy the inhomogeneous Eq. (11) for both finite and infinite $N$, we have to take a convex combination of the two solutions,

$$
\left\langle\sigma_{i} \sigma_{j}\right\rangle=\alpha \sqrt{A_{i} A_{j}} \omega_{-}^{j-i}+\beta \sqrt{A_{i} A_{j}} \omega_{+}^{j-i} \quad \text { with } \alpha+\beta=1,
$$

which then leads to $\gamma_{e} A_{o}+\gamma_{o} A_{e}=\gamma_{e}+\gamma_{o}$. This relation, together with the previous identity $\gamma_{e} A_{o}=\gamma_{o} A_{e}$, determines the values of the two amplitudes $A_{e}$ and $A_{o}$. Demanding periodicity for the ansatz (A3) results in a second identity for the two integration constants $\alpha, \beta$, namely $\beta=\alpha \omega_{-}^{N}$. Comparing the result with our assertion, Eq. (A2), we identify $\omega \equiv \omega_{-}$and $Z(\omega) \equiv 1+\omega_{-}^{N}$. Since $\omega_{-}<1$, the $N \rightarrow \infty$ limit is also obvious.

To treat the general case, we set up some notation. For any even $m \geqslant 2$, we define two auxiliary functions that depend on an ordered set of arguments, $1 \leqslant k_{1}<k_{2}<\cdots<k_{m}$ $\leqslant N$,

$$
\begin{aligned}
& S_{m}^{(f)}\left(k_{1}, k_{2}, \ldots, k_{m}\right) \\
& \equiv \sqrt{A_{k_{1}} A_{k_{2}} \cdots A_{k_{m}}} \omega^{\left(k_{2}-k_{1}\right)+\left(k_{4}-k_{3}\right)+\cdots+\left(k_{m}-k_{m-1}\right)} \\
& =S_{2}^{(f)}\left(k_{1}, k_{2}\right) S_{2}^{(f)}\left(k_{3}, k_{4}\right) \cdots S_{2}^{(f)}\left(k_{m-1}, k_{m}\right), \\
& S_{m}^{(b)}\left(k_{1}, k_{2}, \ldots, k_{m}\right) \\
& \equiv \sqrt{A_{k_{1}} A_{k_{2}} \cdots A_{k_{m}}} \omega^{-\left(k_{2}-k_{1}\right)-\left(k_{4}-k_{3}\right)-\cdots-\left(k_{m}-k_{m-1}\right)} \\
& =S_{2}^{(b)}\left(k_{1}, k_{2}\right) S_{2}^{(b)}\left(k_{3}, k_{4}\right) \cdots S_{2}^{(b)}\left(k_{m-1}, k_{m}\right)
\end{aligned}
$$

Thus, we rewrite Eq. (A2) in the form

$$
\left\langle\sigma_{i} \quad \sigma_{j}\right\rangle=\frac{1}{Z(\omega)}\left[S_{2}^{(f)}(i, j)+\omega^{N} S_{2}^{(b)}(i, j)\right]
$$

and recall that both $S_{2}^{(f)}(i, j)$ and $S_{2}^{(b)}(i, j)$ satisfy the equations of motion for the infinite chain, i.e., Eqs. (10), (11), and (15).

We now proceed as follows: First, we argue that both $S_{m}^{(f)}\left(k_{1}, k_{2}, \ldots, k_{m}\right)$ and $S_{m}^{(b)}\left(k_{1}, k_{2}, \ldots, k_{m}\right)$ solve the homogeneous and inhomogeneous equations of motion (9) for $m$-point functions on the infinite chain. For $S_{m}^{(f)}\left(k_{1}, k_{2}, \ldots, k_{m}\right)=\lim _{N \rightarrow \infty}\left\langle\sigma_{k_{1}} \cdots \sigma_{k_{m}}\right\rangle$, this is immedi- 
ately obvious. For $S_{m}^{(b)}\left(k_{1}, k_{2}, \ldots, k_{m}\right)$, we simply need to retrace the inductive proof from Sec. IV, since the basic relations (10), (11), and (15) all hold for $S_{2}^{(b)}$, we may replace each $\left\langle\sigma_{k_{1}} \cdots \sigma_{k_{m}}\right\rangle$ in Eqs. (17) and (18) by the appropriate $S_{m}^{(b)}$ without violating any of the equalities. Since some of the equations of motion (9) for the $m$-point correlations are inhomogeneous, only a convex combination $\alpha S_{m}^{(f)}\left(k_{1}, k_{2}, \ldots, k_{m}\right)+\beta S_{m}^{(b)}\left(k_{1}, k_{2}, \ldots, k_{m}\right)$ with $\alpha+\beta=1$ is a solution.

Finally, we have to satisfy the remaining property of the full correlation function, namely, periodicity. Given an ordered set of arguments $1 \leqslant k_{1}<k_{2}<\cdots<k_{m} \leqslant N$, we assert that a general correlation function of $m \geqslant 2$ spins takes the form

$$
\begin{aligned}
& \left\langle\sigma_{k_{1}} \cdots \sigma_{k_{m}}\right\rangle=0 \text { for odd } m \text {, } \\
& \left\langle\sigma_{k_{1}} \cdots \sigma_{k_{m}}\right\rangle=\frac{\sqrt{A_{k_{1}} A_{k_{2}} \cdots A_{k_{m}}}}{Z(\omega)}\left\{\omega^{\left(k_{2}-k_{1}\right)+\left(k_{4}-k_{3}\right)+\cdots+\left(k_{m}-k_{m-1}\right)}+\omega^{N-\left[\left(k_{2}-k_{1}\right)+\left(k_{4}-k_{3}\right)+\cdots+\left(k_{m}-k_{m-1}\right)\right]}\right\} \\
& =\frac{1}{Z(\omega)}\left\{S_{m}^{(f)}\left(k_{1}, k_{2}, \ldots, k_{m}\right)+\omega^{N} S_{m}^{(b)}\left(k_{1}, k_{2}, \ldots, k_{m}\right)\right\} \quad \text { for even } m,
\end{aligned}
$$

which is obviously periodic on the ring. Comparing Eqs. (A1) and (A4), it is manifest how closely the correlations of the nonequilibrium model mirror those of the Ising chain. Again, the only significant difference is the appearance of the two amplitudes, $A_{e}$ and $A_{o}$.

[1] E. Ising, Z. Phys. 31, 253 (1925); L. Onsager, Phys. Rev. 65, 117 (1944); Nuovo Cimento, Suppl. 6, 261 (1949); B.M. McCoy and T.T. Wu, The Two-Dimensional Ising Model (Harvard University Press, Cambridge, MA, 1973).

[2] P.L. Garrido, J.L. Lebowitz, C. Maes, and H. Spohn, Phys. Rev. A 42, 1954 (1990); C. Maes, J. Stat. Phys. 61, 667 (1990); Z. Cheng, P.L. Garrido, J.L. Lebowitz, and J.L. Vallés, Europhys. Lett. 14, 507 (1991); C. Maes and F. Redig, J. Phys. I 1, 669 (1991); J. Phys. A 24, 4359 (1991).

[3] T.L. Hill, J. Chem. Phys. 76, 1122 (1982); P.L. Garrido and J. Marro, J. Phys. A 254, 1453 (1992).

[4] H.W.J. Blöte, J.R. Heringa, A. Hoogland, and R.K.P. Zia, J. Phys. A 23, 3799 (1990); Int. J. Mod. Phys. B 5, 685 (1991).

[5] J.-S. Wang, K. Binder, and J.L. Lebowitz, J. Stat. Phys. 56, 783 (1989); M. Droz, Z. Rácz, and J. Schmidt, Phys. Rev. A 39, 2141 (1989).

[6] S. Katz, J.L. Lebowitz, and H. Spohn, Phys. Rev. B 28, 1655 (1983); J. Stat. Phys. 34, 497 (1984).

[7] B. Schmittmann and R.K.P. Zia, Statistical Mechanics of Driven Diffusive Systems, edited by C. Domb and J.L. Lebowitz, Phase Transitions and Critical Phenomena Vol. 17 (Academic Press, London, 1995).

[8] For reviews, see, e.g., R. Schmitz, Phys. Rep. 171, 1 (1988); J.R. Dorfman, T.R. Kirkpatrick, and J.V. Sengers, Annu. Rev. Phys. Chem. 45, 213 (1994).

[9] F. Bonetto, J.L. Lebowitz, and L. Rey-Bellet, Fourier Law: A Challenge to Theorists, in Mathematical Physics 2000 (Imperial College Press, London, 2000).

[10] H. Spohn, J. Phys. A 16, 4275 (1983); H.C. Fogedby and A. Svane, Phys. Rev. B 42, 1056 (1990); J.V. Andersen and O.G. Mouritsen, Phys. Rev. Lett. 65, 440 (1990).

[11] B. Schmittmann and R.K.P. Zia, Phys. Rev. Lett. 66, 357
(1991); B. Schmittmann, Europhys. Lett. 24, 109 (1993).

[12] H.K. Janssen and B. Schmittmann, Z. Phys. B: Condens. Matter 64, 503 (1986); K.t. Leung and J.L. Cardy, J. Stat. Phys. 44, 567 (1986); 45, 1087(E) (1986).

[13] U.C. Täuber, V.K. Akkineni, and J.E. Santos, Phys. Rev. Lett. 88, 045702 (2002).

[14] G. Grinstein, C. Jayaprakash, and Y. He, Phys. Rev. Lett. 55, 2527 (1985); K.E. Bassler and B. Schmittmann, ibid. 73, 3343 (1994).

[15] F. Schmüser and B. Schmittmann, J. Phys. A 35, 2569 (2002).

[16] R.J. Glauber, J. Math. Phys. 44, 294 (1963); B.U. Felderhof, Rep. Math. Phys. 1, 215 (1971); 2, 151 (1971).

[17] Z. Racz and R.K.P. Zia, Phys. Rev. E 49, 139 (1993).

[18] G. Schütz, Exactly Solvable Models for Many-body Systems Far from Equilibrium, edited by C. Domb and J.L. Lebowitz, Phase Transitions and Critical Phenomena Vol. 19 (Academic Press, London, 2000).

[19] Nonequilibrium Statistical Mechanics in One Dimension, edited by V. Privman (Cambridge University Press, Cambridge, England, 1997).

[20] B. Derrida, J.L. Lebowitz, and E.R. Speer, Phys. Rev. Lett. 87, 150601 (2001); 89, 030601 (2002).

[21] M. Hastings and E. Ben-Naim (private communication).

[22] F. Schmüser and W. Just, J. Stat. Phys. 105, 525 (2001).

[23] R.K.P. Zia and T. Blum, in Scale Invariance, Interfaces and Non-equilibrium Dynamics, edited by M. Droz, A.J. McKane, J. Vannimenus, and D.E. Wolf (Plenum, New York, 1995); R.K.P. Zia, L.B. Shaw, B. Schmittmann, and R.J. Astalos, Comput. Phys. Commun. 127, 23 (2000).

[24] D.E. Bedeaux, K.E. Shuler, and I. Oppenheim, J. Stat. Phys. 2, 1 (1970).

[25] H.J. Hilhorst (private communication).

[26] H.J. Hilhorst, B. Schmittmann, and R.K.P. Zia (unpublished). 